International Journal of Pure and Applied Mathematics

Volume 115 No. 1 2017, 187-198

ISSN: 1311-8080 (printed version); ISSN: 1314-3395 (on-line version)

url: http://www.ijpam.eu

doi: 10.12732/ijpam.v115i1.14

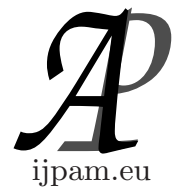

\title{
ANALYTICAL SOLUTIONS OF THE IVANCEVIC OPTION PRICING MODEL WITH A NONZERO ADAPTIVE MARKET POTENTIAL
}

\author{
S.O. Edeki ${ }^{1}$, O.O. Ugbebor ${ }^{2}$, O. González-Gaxiola ${ }^{3}$ \\ ${ }^{1,2}$ Department of Mathematics \\ Covenant University \\ Canaanland, Ota, NIGERIA \\ ${ }^{2}$ Department of Mathematics \\ University of Ibadan \\ Ibadan, NIGERIA \\ ${ }^{3}$ Departamento de Matemáticas Aplicadas y Sistemas \\ Universidad Autónoma Metropolitana-Cuajimalpa \\ Vasco de Quiroga 4871, Cuajimalpa, 05348, MEXICO
}

\begin{abstract}
The Ivancevic option pricing model (IOPM) is a nonlinear adaptive-wave alternative for the classical Black-Scholes option pricing model, representing a controlled Brownian motion in an adaptive setting relating to nonlinear Schrödinger equation. Despite the applicability and usefulness of the IOPM, analytical solutions of such model are barely found in literature. Therefore, this paper obtains analytical solutions of the IOPM by means of a proposed semi-analytical method referred to as projected differential transform method (PDTM). Cases of nonzero adaptive market potential are considered. The proposed method is proven to be direct, and effective as the obtained solutions tend rapidly to their exact forms.
\end{abstract}

AMS Subject Classification: 34G20, 93E35, 81Q05, 97M30

Key Words: Ivancevic pricing model, nonlinear Black-Scholes model, option pricing, PDTM

Received: $\quad$ May 12, 2017

Revised: June 20, 2017

Published: June 29, 2017

(c) 2017 Academic Publications, Ltd. url: www.acadpubl.eu

$\S_{\text {Correspondence author }}$ 


\section{Introduction}

In mathematical finance, the classical Black-Scholes model serves as hallmark financial model; it describes the time-evolution of the market value of financial equity such as stock option $[1,2,3]$. The basic assumptions under which this classical arbitrage pricing theory is formulated include the following: the asset price $S$ (or the underlying asset) following a geometric Brownian motion $(\mathrm{GBM})$, the drift parameter, $\mu$ and the volatility rate, $\sigma$ are assumed constants, lack of arbitrage opportunities (no risk-free profit), frictionless and competitive markets $[4,5,6]$. Thus, the stock price $S=S(t)$, at time $t,(0 \leq t \leq T)$ follows the stochastic differential equation (SDE):

$$
d S=S\left(\mu d t+\sigma d W_{t}\right), S \in[0, \infty)
$$

where $\mu, \sigma>0$, and $W_{t}$ are mean rate of return of $S$, the volatility, and a standard Brownian motion respectively.

So, for an option value $u=u(S, t)$, the Black-Scholes partial differential equation (PDE) associated to (1.1) can be expressed as:

$$
\frac{\partial u}{\partial t}+r S \frac{\partial u}{\partial S}+\frac{1}{2} S^{2} \sigma^{2} \frac{\partial^{2} u}{\partial S^{2}}-r u=0
$$

with $u(0, t)=0, u(S, t) \rightarrow 0$ as $S \rightarrow \infty, u(S, T)=\max (S-E, 0), E$ is a constant and

$$
S(t)=S_{0} e^{\left(\mu-\frac{\sigma^{2}}{2}\right) t+\sigma W_{t}}, \quad S_{0}=S(0) .
$$

In literature, detailed and extensive work on the importance of (1.2) with respect to exact, analytical, approximate or numerical methods of solutions have been captured $[7,8,9,10]$.

Recently, Vukovic [11], established the interconnectedness of the Schrödinger and the Black-Scholes equations via the tools of quantum physics in the sense of Hamiltonian operator. It was noted that while the Black-Scholes Hamiltonian was anti-Hermitian causing the eigenvalues to be complex, the Schrödinger Hamiltonian was Hermitian. It was further showed that the Black-Scholes equation can be derived from the Schrödinger equation via the application of quantum mechanics tools $[12,13]$. The facts incorporated include the points that: the Schrödinger equation requires a complex state function while the BlackScholes equation is a real PDE that yields a real valued expression for the option price at all time.

The Black-Scholes model (1.2) can be applied to a reasonable number of one dimensional option models ascribed to $u$ and $S$, say for puts/calls and stocks/dividends respectively [2]. As noted in $[14,15]$, one could consider the associated 
probability density function (PDF) resulting from the backward Fokker-Planck equation using the classical Kolmogorov probability method instead of the market value of an option obtained via the Black-Scholes equation.

\section{The Ivancevic Option Pricing Model (IOPM) [16]}

As an alternative method for obtaining the same PDF for the market value of a stock option, Ivancevic [17] applied the quantum-probability formation as a solution to a time-dependent Schrödinger equation (linear or nonlinear) for the evolution of the complex-valued wave function, and proposed an adaptive, wave-form nonlinear model $[18,19]$. Henceforth, such nonlinear adaptive model is referred to as Ivancevic option pricing model as follows:

$$
i \frac{\partial w}{\partial t}+\frac{1}{2} \sigma^{2} \frac{\partial^{2} w}{\partial S^{2}}+\beta|w|^{2} w=0, \quad i^{2}=-1
$$

where $w=w(S, t)$ denotes the option pricing wave-function at time $t,|w|^{2}=$ $|w(S, t)|^{2}$ represents the PDF for the option price with regard to stock price and time, $\sigma$ represents a constant or stochastic process as the dispersion frequency volatility coefficient while $\beta$ is referred to as the Landau coefficient representing adaptive market potential. The model (2.1) becomes linear if $\beta=0$. In this work, a case of non-zero adaptive market potential $(\beta \neq 0)$ will be considered in terms of analytical solutions using a proposed semi-analytical method referred to as projected differential transformation method (PDTM).

\section{The Overview of the PDT Method [20, 21]}

In this section, an overview of the PDTM is being presented.

\subsection{A Note on Some Basic Theorems of the PDTM}

In consideration, let $u(x, t)$ be an analytic function at $\left(x_{*}, t_{*}\right)$ in a domain $D$, then in considering the Taylor series of $u(x, t)$, we give regard to some variables $s_{v}=t$ instead of all the variables as seen in the classical DTM. Thus, the projected DTM of $u(x, t)$ with respect to $t$ at $t_{*}$ is defined and denoted by:

$$
U(x, h)=\frac{1}{h !}\left[\frac{\partial^{h} u(x, t)}{\partial t^{h}}\right]_{t=t_{*}}
$$


such that

$$
U(x, h)=\sum_{h=0}^{\infty} U(x, h)\left(t-t_{*}\right)^{h}
$$

where (3.2) is referred to as the projected differential inverse transform (PDIT) of with respect to the time parameter $t$.

\subsection{Some Basic Properties and Theorems of the PDTM}

(a) If $m(x, t)=\alpha m_{a}(x, t)+\beta m_{b}(x, t)$, then $M(x, h)=\alpha M_{a}(x, h)+\beta M_{b}(x, h)$.

(b) If $m(x, t)=\alpha \frac{\partial^{n} m_{*}(x, t)}{\partial t^{n}}$, then $M(x, h)=\alpha \frac{(h+n) !}{h !} M_{*}(x, h+n)$.

(c) If $m(x, t)=\alpha \frac{\partial m_{*}(x, t)}{\partial t}$, then $M(x, h)=\alpha \frac{(h+1) !}{h !} M_{*}(x, h+1)$.

(d) If $m(x, t)=p(x) \frac{\partial^{n} m_{*}(x, t)}{\partial x^{n}}$, then $M(x, h)=p(x) \frac{\partial^{n} M_{*}(x, h)}{\partial x^{n}}$.

(e) If $m(x, t)=p(x) m_{*}^{2}(x, t)$, then $M(x, h)=p(x) \sum_{r=0}^{h} M_{*}(x, r) M_{*}(x, h-r)$.

(f) If $p(x, y)=x^{r} y^{r^{*}}$, then $P(k, h)=\delta\left(k-r, h-r^{*}\right)=\delta(k-r) \delta\left(h-r^{*}\right)$,

where $\delta$ is the Kronecker delta. Thus, $u(x, t)=\sum_{h=0}^{\infty} U(x, h) t^{h}$.

\section{The PDTM and the Ivancevic Option Pricing Model}

In this subsection, the PDTM will be applied to the model equation (2.1) as follows. According to [16], (2.1) will be subjected to a plane function, $w(S, 0)=$ $f(S)$ as an initial condition, thereby yielding the following initial value problem (IVP):

$$
\left\{\begin{array}{l}
i \frac{\partial w}{\partial t}+\frac{1}{2} \sigma^{2} \frac{\partial^{2} w}{\partial S^{2}}+\beta|w|^{2} w=0 \\
w(S, 0)=f(S)
\end{array}\right.
$$

where $w=w(S, t)$ denotes the option pricing wave function at time $t, \sigma$ represents a constant or stochastic process as the dispersion frequency volatility coefficient while $\beta$ is referred to as the Landau coefficient representing adaptive market potential.

For simplicity, we re-expressed (4.1) as:

$$
\left\{\begin{array}{l}
\frac{\partial w}{\partial t}=i\left(\frac{1}{2} \sigma^{2} \frac{\partial^{2} w}{\partial S^{2}}+\beta|w|^{2} w\right) \\
w(S, 0)=f(S)
\end{array}\right.
$$

Transforming (4.2) using PDTM yields:

$$
\left\{\begin{array}{l}
(k+1) W(S, k+1)=i\left(\frac{1}{2} \sigma^{2} W_{S S}(S, k)+\beta H(s)\right), \\
W(S, 0)=f(S) .
\end{array}\right.
$$


where $\bar{W}(\cdot)$ denotes the complex conjugate of $W(\cdot)$, and

$$
H(s)=\sum_{r=0}^{k} \sum_{n=0}^{k-r} \bar{W}(S, r) W(S, n) W(S, k-r-n) .
$$

This implies that:

$$
\left\{\begin{array}{l}
W(S, k+1)=\frac{i}{(k+1)}\left(\frac{1}{2} \sigma^{2} W_{S S}(S, k)+\beta H(s)\right) \\
W(S, 0)=w(S, 0)
\end{array}\right.
$$

Therefore, for $W(S, \zeta)=W(\zeta)$ and, $k=0,1,2,3, \ldots$, the following are respectively obtained:

$$
\left\{\begin{array}{l}
W(1)=i\left(\frac{1}{2} \sigma^{2} W_{S S}(0)+\beta \bar{W}(0) W^{2}(0)\right) \\
W(2)=\frac{i}{2}\left(\frac{1}{2} \sigma^{2} W_{S S}(1)+\beta\left(2 \bar{W}(0) W(0) W(1)+\bar{W}(1) W^{2}(0)\right)\right),
\end{array}\right.
$$

$$
\begin{aligned}
W(3)= & \frac{i}{3}\left(\frac{1}{2} \sigma^{2} W_{S S}(2)+\beta\left(2 \bar{W}(0) W(0) W(2)+\bar{W}(0) W^{2}(1)+2 \bar{W}(1) W(0) W(1)\right.\right. \\
& \left.\left.+\bar{W}(1) W^{2}(0)\right)\right), \\
W(4)= & \frac{i}{4}\left(\frac{1}{2} \sigma^{2} W_{S S}(3)+\beta(2 \bar{W}(0) W(0) W(3)+2 \bar{W}(0) W(1) W(2)\right. \\
& \left.\left.+2 \bar{W}(1) W(0) W(2)+\bar{W}(1) W^{2}(1)+2 \bar{W}(2) W(0) W(1)+\bar{W}(3) W^{2}(0)\right)\right), \\
W(5)= & \frac{i}{5}\left(\frac{1}{2} \sigma^{2} W_{S S}(4)+\beta\left(2 \bar{W}(0) W(0) W(4)+2 \bar{W}(0) W(1) W(3)+\bar{W}(0) W^{2}(2)\right.\right. \\
& +2 \bar{W}(1) W(0) W(3)+2 \bar{W}(1) W(1) W(2)+2 \bar{W}(2) W(0) W(2) \\
& \left.\left.+\bar{W}(2) W^{2}(1)+2 \bar{W}(3) W(0) W(1)+\bar{W}(4) W^{2}(0)\right)\right),
\end{aligned}
$$

and so on.

\subsection{Numerical Illustrative Examples}

In this subsection, we consider the following cases for numerical computation:

Case A: Suppose $\beta=2, \sigma=\sqrt{2}$ and $f(S)=e^{2 i S}$. Then the corresponding Ivancevic option pricing model is:

$$
\left\{\begin{array}{l}
\frac{\partial w}{\partial t}=i\left(\frac{\partial^{2} w}{\partial S^{2}}+2|w|^{2} w\right) \\
w(S, 0)=e^{2 i S}
\end{array}\right.
$$


So, applying the PDTM with the parameters in case A through (4.4) gives the following:

$$
\begin{gathered}
W(0)=e^{2 i S}, \quad W(1)=-2 i e^{2 i S}, \quad W(2)=-2 e^{2 i S}, \quad W(3)=\frac{4 i}{3} e^{2 i S}, \\
W(4)=\frac{2}{3} e^{2 i S}, \quad W(5)=-\frac{4 i}{15} e^{2 i S}, \quad W(6)=-\frac{4}{45} e^{2 i S}, \quad W(7)=\frac{8 i}{315} e^{2 i S}, \\
W(8)=\frac{2}{315} e^{2 i S}, \quad W(9)=-\frac{4 i}{2835} e^{2 i S}, \text { and so on. }
\end{gathered}
$$

Whence,

$$
\begin{aligned}
w(S, t) & =\sum_{n=0}^{\infty} W(n) t^{n} \\
& =W(0)+W(1) t+W(2) t^{2}+W(3) t^{3}+W(4) t^{4}+\cdots \\
& =e^{2 i S}\left(1-2 i t-2 t^{2}+\frac{4 i}{3} t^{3}+\frac{2}{3} t^{4}-\frac{4 i}{15} t^{5}-\frac{4}{45} t^{6}+\frac{8 i}{315} t^{7}+\frac{2}{315} t^{8}-\cdots\right) \\
& =e^{2 i S}\left(1+(-2 i t)+\frac{(-2 i t)^{2}}{2 !}+\frac{(-2 i t)^{3}}{3 !}+\frac{(-2 i t)^{4}}{4 !}+\frac{(-2 i t)^{5}}{5 !}+\cdots\right) \\
& =e^{2 i S} e^{-2 i t} \\
& =\cos 2(S-t)+i \sin 2(S-t) .
\end{aligned}
$$

Showing that

$$
w(S, t)=e^{2 i(S-t)}
$$

satisfies (4.5) is obvious and straightforward.

Case B: Suppose $\beta=6, \sigma=\sqrt{2}$ and $f(S)=1$. Then the corresponding Ivancevic option pricing model is:

$$
\left\{\begin{array}{l}
\frac{\partial w}{\partial t}=i\left(\frac{\partial^{2} w}{\partial S^{2}}+6|w|^{2} w\right) \\
w(S, 0)=1
\end{array}\right.
$$

with an exact solution:

$$
w(S, t)=e^{6 i t}
$$

So, applying the PDTM with the parameters in case A through (4.4) gives the following:

$$
W(0)=1, \quad W(1)=6 i, \quad W(2)=-18, \quad W(3)=-36 i, \quad W(4)=54,
$$


Table 1: Solutions of Case A (real parts).

\begin{tabular}{|l|l|r|r|}
\hline$t$ & $R e\left(w_{e x}\right)$ & $R e\left(w_{P D T M}\right)$ & Rel. error \\
\hline 0.5 & 0.540302 & 0.5393051 & 0.001845079 \\
\hline 0.6 & 0.696707 & 0.6955375 & 0.001678611 \\
\hline 0.7 & 0.825336 & 0.8240410 & 0.001569058 \\
\hline 0.8 & 0.921061 & 0.9196927 & 0.001485569 \\
\hline 0.9 & 0.980067 & 0.9786790 & 0.001416230 \\
\hline 1.0 & 1.000000 & 0.9986486 & 0.001351400 \\
\hline 1.1 & 0.980067 & 0.9788051 & 0.001287565 \\
\hline 1.2 & 0.921061 & 0.9199398 & 0.001217292 \\
\hline 1.3 & 0.825336 & 0.8243994 & 0.001134811 \\
\hline 1.4 & 0.696707 & 0.6959928 & 0.001025108 \\
\hline 1.5 & 0.540302 & 0.5398392 & 0.000856558 \\
\hline
\end{tabular}

$$
\begin{gathered}
W(5)=\frac{324 i}{5}, \quad W(6)=-\frac{324}{5}, \quad W(7)=-\frac{1944 i}{35}, \quad W(8)=\frac{1458}{35} \\
W(9)=\frac{972 i}{35}, \quad W(10)=-\frac{2916}{175}, \quad W(11)=-\frac{17496 i}{1925}, \quad W(12)=\frac{8748}{1925}
\end{gathered}
$$

and so on. Whence,

$$
\begin{aligned}
w(S, t) & =\sum_{n=0}^{\infty} W(n) t^{n} \\
& =W(0)+W(1) t+W(2) t^{2}+W(3) t^{3}+W(4) t^{4}+\cdots \\
& =\left(1-18 t^{2}+54 t^{4}-\frac{324}{5} t^{6}+\frac{1458}{35} t^{8}-\frac{2916}{175} t^{10}+\frac{8748}{1925} t^{12}+\cdots\right) \\
& +i\left(6 t-36 t^{3}+\frac{324}{5} t^{5}-\frac{1944}{35} t^{7}+\frac{972}{35} t^{9}-\frac{17946}{1925} t^{11}+\frac{52488}{25025} t^{13}+\cdots\right) .
\end{aligned}
$$

Remark: The results of Case A and Case B are presented in Tables 1-4 below. We use $\operatorname{Re}\left(w_{\text {ex }}\right), \operatorname{Re}\left(w_{P D T M}\right), \operatorname{Im}\left(w_{\text {ex }}\right), \operatorname{Im}\left(w_{P D T M}\right)$, and Rel. error to denote real part of the exact solution, real part of the approximate solution, imaginary part of the exact solution, imaginary part of the approximate solution, and relative error respectively. In Figure 1 and Figure 2, the real, and the imaginary parts associated to the solution of Case A are graphically presented respectively. 
Table 2: Solutions of Case A (imaginary parts).

\begin{tabular}{|l|l|r|r|}
\hline$t$ & $\operatorname{Im}\left(w_{e x}\right)$ & $\operatorname{Im}\left(w_{P D T M}\right)$ & Rel. error \\
\hline 1.1 & 0.198669 & 0.198090 & 0.002915 \\
\hline 1.2 & 0.389418 & 0.388600 & 0.002101 \\
\hline 1.3 & 0.564642 & 0.563618 & 0.001814 \\
\hline 1.4 & 0.717356 & 0.716166 & 0.001660 \\
\hline 1.5 & 0.841471 & 0.840162 & 0.001555 \\
\hline 1.6 & 0.932039 & 0.930665 & 0.001475 \\
\hline 1.7 & 0.985450 & 0.984064 & 0.001406 \\
\hline 1.8 & 0.999574 & 0.998232 & 0.001343 \\
\hline 1.9 & 0.973848 & 0.972604 & 0.001278 \\
\hline 2.0 & 0.909297 & 0.908201 & 0.001206 \\
\hline
\end{tabular}

Table 3: Solutions of Case B (real parts).

\begin{tabular}{|l|l|r|r|}
\hline$t$ & $R e\left(w_{e x}\right)$ & $R e\left(w_{P D T M}\right)$ & Rel. error \\
\hline 0.01 & 0.059964 & 0.0599640 & 0.000000000 \\
\hline 0.02 & 0.992809 & 0.9928086 & $4.02897 \mathrm{E}-07$ \\
\hline 0.03 & 0.983844 & 0.9838437 & $3.04926 \mathrm{E}-07$ \\
\hline 0.04 & 0.971338 & 0.9713380 & 0.000000000 \\
\hline 0.05 & 0.955336 & 0.9553365 & $5.23376 \mathrm{E}-07$ \\
\hline 0.06 & 0.935897 & 0.9358968 & $2.13699 \mathrm{E}-07$ \\
\hline 0.07 & 0.913089 & 0.9130889 & $1.09518 \mathrm{E}-07$ \\
\hline 0.08 & 0.886995 & 0.8869949 & $1.12740 \mathrm{E}-07$ \\
\hline 0.09 & 0.857709 & 0.8577087 & $3.49769 \mathrm{E}-07$ \\
\hline 0.10 & 0.825336 & 0.8253356 & $4.84651 \mathrm{E}-07$ \\
\hline 0.11 & 0.789992 & 0.7899922 & $2.53167 \mathrm{E}-07$ \\
\hline 0.12 & 0.751806 & 0.7518057 & $3.99039 \mathrm{E}-07$ \\
\hline 0.13 & 0.710914 & 0.7109135 & $7.03320 \mathrm{E}-07$ \\
\hline 0.14 & 0.667463 & 0.6674628 & $2.99642 \mathrm{E}-07$ \\
\hline 0.15 & 0.621610 & 0.6216100 & 0.000000000 \\
\hline
\end{tabular}




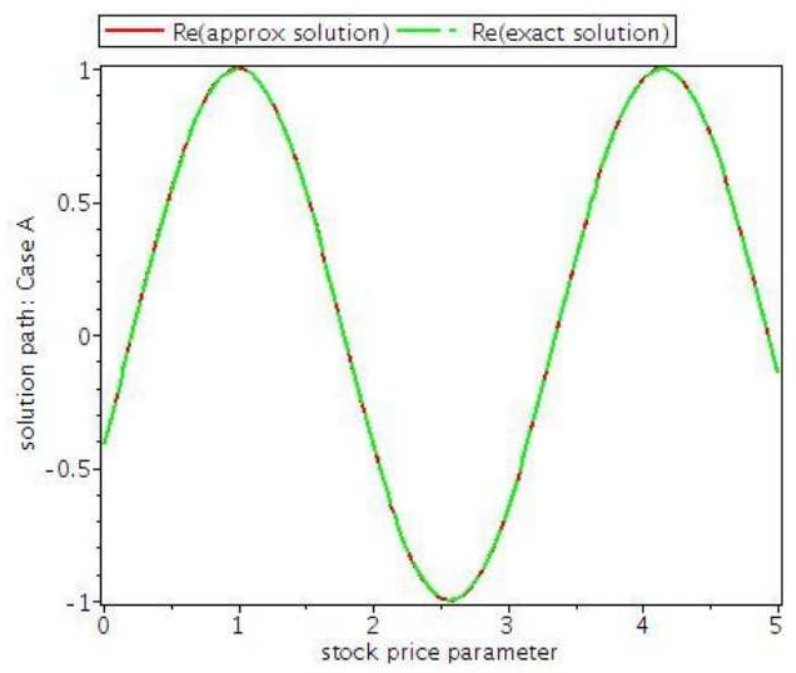

Figure 1: Solution graph of real part content of Case A

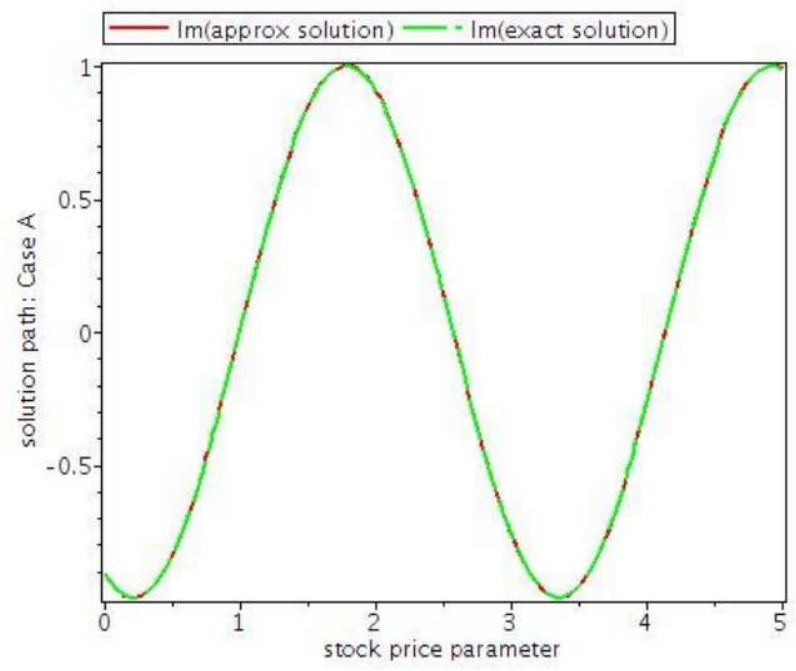

Figure 2: Solution graph of imaginary part content of Case A

\section{Concluding Remarks}

In this paper, we considered the Ivancevic option pricing model (IOPM). This nonlinear adaptive-wave model serves as alternative for the classical Black- 
Table 4: Solutions of Case B (imaginary parts).

\begin{tabular}{|l|l|r|r|}
\hline$t$ & $R e\left(w_{e x}\right)$ & $R e\left(w_{P D T M}\right)$ & Rel. error \\
\hline 0.01 & 0.059964 & 0.059964 & 0.000000 \\
\hline 0.02 & 0.119712 & 0.119712 & $1.67 \mathrm{E}-06$ \\
\hline 0.03 & 0.179030 & 0.179030 & $2.23 \mathrm{E}-06$ \\
\hline 0.04 & 0.237703 & 0.237703 & $1.68 \mathrm{E}-06$ \\
\hline 0.05 & 0.295520 & 0.295520 & $6.77 \mathrm{E}-07$ \\
\hline 0.06 & 0.352274 & 0.352274 & $5.68 \mathrm{E}-07$ \\
\hline 0.07 & 0.407760 & 0.407761 & $1.23 \mathrm{E}-06$ \\
\hline 0.08 & 0.461779 & 0.461779 & $4.33 \mathrm{E}-07$ \\
\hline 0.09 & 0.514136 & 0.514136 & 0.000000 \\
\hline 0.10 & 0.564642 & 0.564643 & $8.86 \mathrm{E}-07$ \\
\hline 0.11 & 0.613117 & 0.613117 & $1.63 \mathrm{E}-07$ \\
\hline 0.12 & 0.659385 & 0.659385 & $4.55 \mathrm{E}-07$ \\
\hline 0.13 & 0.703279 & 0.703279 & $5.69 \mathrm{E}-07$ \\
\hline 0.14 & 0.744643 & 0.744643 & $1.34 \mathrm{E}-07$ \\
\hline 0.15 & 0.783327 & 0.783327 & $1.28 \mathrm{E}-07$ \\
\hline
\end{tabular}

Scholes option pricing model based on a controlled Brownian motion in an adaptive setting relating to nonlinear Schrdinger equation. By considering cases of nonzero adaptive market potential, analytical solutions of the IOPM by means of a proposed semi-analytical method (projected differential transform method) were obtained. Based on the results, the proposed method is proven to be direct, and effective as the obtained solutions tend rapidly to their exact forms without any form of linearization, perturbation, or discretization.

\section{Acknowledgements}

The authors Edeki and Ugbebor are sincerely grateful to Covenant University for the provision of resources, and enabling working environment. They also wish to thank the anonymous referee(s) for their constructive and helpful comments. 


\section{References}

[1] F. Black, M. Scholes, The pricing options and corporate liabilities, J. Political Econ., 81 (1973), 637-654.

[2] V. G. Ivancevic, Adaptive Wave Models for Sophisticated Option Pricing, Journal of Mathematical Finance, 1 (2011), 41-49.

[3] R. C. Merton, Theory of Rational Option Pricing, The Bell Journal of Economics and Management Science, 4 (1973), 141-183, doi: 10.2307/3003143.

[4] O. González-Gaxiola, J. Ruíz de Chávez and J. A. Santiago, A Nonlinear Option Pricing Model Through the Adomian Decomposition Method, Int. J. Appl. Comput. Math., 2 (2016), 453-467, doi: 10.1007/s40819-015-0070-6.

[5] S. O. Edeki, E. A. Owoloko, O. O. Ugbebor, The Modified Black-Scholes Model via Constant Elasticity of Variance for Stock Options Valuation, AIP Conference proceedings, 1705 (2016), 020041, doi: 10.1063/1.4940289.

[6] G. Barles, H. M. Soner, Option pricing with transaction costs and a nonlinear BlackScholes equation, Financ. Stoch., 2 (1998), 369-397.

[7] M. R. Rodrigo, R. S. Mamon, An alternative approach to solving the Black-Scholes equation with time-varying parameters, Appl. Math. Lett., 19 (2006), 398-402.

[8] S. O. Edeki, O. O. Ugbebor, E. A. Owoloko, Analytical Solutions of the Black-Scholes Pricing Model for European Option Valuation via a Projected Differential Transformation Method, Entropy, 17 (2015), 7510-7521.

[9] R. Company, E. Navarro, J. R. Pintos, E. Ponsoda, Numerical solution of linear and nonlinear Black-Scholes option pricing equations, Comput. Math. Appl., 56 (2008), 813821.

[10] S. O. Edeki, O. O. Ugbebor, E. A. Owoloko, He's Polynomials for Analytical Solutions of the Black-Scholes Pricing Model for Stock Option Valuation, Lecture Notes in Engineering and Computer Science: Proceedings of the World Congress on Engineering 2016, WCE 2016, June 29 - July 1, London, U.K., (2016), 632-63.

[11] O. Vukovic, On the Interconnectedness of Schrödinger and Black-Scholes Equation, Journal of Applied Mathematics and Physics, 3 (2015), 1108-1113.

[12] B. E. Baaquie, Quantum Finance: Path Integrals and Hamiltonians for Options and Interest Rates. Cambridge University Press, Cambridge, (2004).

[13] M. Contreras, R. Pellicer, M. Villena, A. Ruiz, A Quantum Model of Option Pricing: When Black-Scholes meets Schrödinger and Its Semi-Classical Limit, Physica A, 389 (2010), 5447-5459.

[14] C. W. Gardiner, Handbook of Stochastic Methods, Springer Berlin, (1983).

[15] J. Voit, The Statistical Mechanics of Financial Markets, Springer, Berlin, (2005).

[16] O. González-Gaxiola, and J. Ruíz de Chávez, Solving the Ivancevic option pricing model using the Elsaki-Adomian decomposition method, Int. J. of Applied Math., 28 (2015), 515-525.

[17] V. Ivancevic, Adaptive-Wave Alternative for the Black-Scholes Option Pricing Model, Cognitive Computation, 2 (2010), 17-30.

[18] R. Cont, Empirical properties of asset returns: stylized facts and statistical issues, Quant. Finance 1 (2001), 223-236. 
[19] J. Perello, R. Sircar, J. Masoliver, Option pricing under stochastic volatility: the exponential Ornstein-Uhlenbeck model, J. Stat. Mech. P06010, (2008).

[20] S. O. Edeki, G. O. Akinlabi, S. A. Adeosun, On a modified transformation method for exact and approximate solutions of linear Schrödinger equations, AIP Conference Proceedings, 1705, 020048 (2016), doi: 10.1063/1.4940296.

[21] A. S. V. Ravi Kanth, K. Aruna, Comparison of two dimensional DTM and PTDM for solving time-dependent Emden-Fowler Type Equations, Int. J. of Nonlinear Science, 13 (2012), 228-239. 